\title{
De autonomia roubada ao fortalecimento da profissão: conexões entre Enfermagem Brasileira e Africana
}

\author{
From stolen autonomy to the strengthening of the profession: connections between the Brazilian and \\ African Nursing \\ De la autonomía robada al fortalecimiento de la profesión: conexiones entre Enfermería Brasileña y \\ Africana
}

\author{
Carla Aparecida Arena Ventura ${ }^{1}$ (1) \\ Isabel Amélia Costa Mendes ${ }^{1}$ (B) \\ Ítalo Rodolfo Silva² (1) \\ Leila Maria Marchi-Alves ${ }^{1}$ (D) \\ Emerson Willian Santos Almeida ${ }^{1}$ (D) \\ Simone de Godoy ${ }^{1}$ (10)
}

1. Universidade de São Paulo, Escola de Enfermagem de Ribeirão Preto. Ribeirão Preto, SP, Brasil.

2. Universidade Federal de Rio de Janeiro. Campus Macaé, Macaé, RJ. Brasil.

Autor correspondente:

Carla Aparecida Arena Ventura.

E-mail: caaventu@eerp.usp.br.

Submitted on $08 / 04 / 2020$.

Accepted on $11 / 03 / 2020$.

DOI:https://doi.org/10.1590/2177-9465-EAN-2020-0265

\section{Resumo}

Objetivo: caracterizar os desafios vivenciados pela Enfermagem nos países africanos de língua portuguesa e discutir as potencialidades da cooperação internacional neste contexto. Método: estudo qualitativo, descritivo. Dados obtidos por meio de entrevista semiestruturada com enfermeiros de países africanos de língua portuguesa e categorizados por análise de conteúdo. Resultados: participaram nove enfermeiros, provenientes dos seguintes países: Angola, Cabo Verde, Guiné-Bissau, Moçambique e São Tomé e Príncipe. Foram desenvolvidas duas categorias: a primeira retrata as dificuldades da Enfermagem de países africanos de língua portuguesa acerca da autonomia e valorização necessárias ao desenvolvimento e exercício de suas competências. A segunda categoria evidenciou possibilidades de conexões a partir de redes para integração e fortalecimento da Enfermagem, em cooperação internacional. Conclusão e implicações para a prática: o estudo caracterizou como principais dificuldades aquelas relacionadas a processos de trabalho, falta de autonomia, de estrutura humana e física. Nessa perspectiva, a cooperação internacional representou importante estratégia para o fortalecimento da formação dos enfermeiros de países africanos de língua portuguesa, assim como para lidarem com os expressivos desafios que se apresentam em sua prática cotidiana.

Palavras-chave: Cooperação Internacional; Comunidade dos Países de Língua Portuguesa; Enfermagem, Autonomia Profissional; Recursos Humanos, Enfermagem.

\section{Abstract}

Aim: to characterize the challenges experienced by nurses in Portuguese speaking African countries and discuss the potential for international cooperation in this context. Method: qualitative and descriptive study. Data were collected through semi-structured interviews with nurses from Portuguese speaking African countries and categorized through content analysis. Results: nine nurses participated in the study. They were from the following countries: Angola, Cape Verde, Guinea Bissau, Mozambique and San Tome and Prince. Two categories emerged from the results, one focusing on the difficulties of Nursing in Portuguese speaking African countries regarding their autonomy and the need to value the development and exercise of their competencies. The second evidenced possibilities of connections based on networks for the integration and strengthening of Nursing, through international cooperation. Conclusion and implications for practice: the study characterized as main difficulties the ones related to the work process, lack of autonomy, of human and physical structure. Therefore, international cooperation emerged as an important strategy for the capacity building of nurses from Portuguese-speaking African countries, as well as to deal with the huge challenges faced by nurses in their daily activities.

Keywords: International Cooperation; Community of Portuguese-speaking countries; Nursing, Professional Autonomy; Human Resources, Nursing

\section{REsumen}

Objetivo: caracterizar los retos vivenciados por la Enfermería en los países africanos de lengua portuguesa y discutir las potencialidades de la cooperación internacional en este contexto. Método: estudio cualitativo y descriptivo. Datos recolectados por medio de entrevistas semiestructuradas con enfermeros de países africanos de lengua portuguesa y categorizados por medio de análisis de contenido. Resultados: participaron nueve enfermeros de los países: Angola, Cabo Verde, Guiné-Bissau, Mozambique y San Tomé y Príncipe. Fueron desarrolladas dos categorías, una que retrata las dificultades de la Enfermería en los países africanos de lengua portuguesa sobre la autonomía y apreciación necesárias al desarrollo y ejercício de sus competencias. La segunda ha evidenciado posibilidades de conexiones a partir de redes para integración y fortalecimiento de la Enfermería, por medio de la cooperación internacional. Conclusión e implicaciones para la práctica: el estudio ha caracterizado como principales dificultades las relacionadas a procesos de trabajo, falta de autonomía, de estructura humana y física. En esa perspectiva, la cooperación internacional ha representado importante estratégia para el fortalecimiento de la formación de enfermeros de países africanos de lengua portuguesa, así como para lidiar con los expresivos retos que se presentan en su práctica cotidiana.

Palabras clave: Cooperación Internacional; Comunidad de Países de Lengua Portuguesa; Enfermería, Autonomía Profesional; Recursos Humanos, Enfermería. 


\section{INTRODUÇÃO}

O Relatório sobre o Estado da Enfermagem no Mundo aponta que $81 \%$ dos profissionais de enfermagem residem em países das regiões das Américas, da Europa e do Pacífico Ocidental; por outro lado, existe um desnível quantitativo de enfermeiros por habitantes nas regiões da África, Sudeste Asiático, Mediterrâneo Oriental e parte da América Latina, sendo destacada a desigualdade relacionada ao reduzido poder econômico desses países. Ressalte-se, contudo, que de cada oito enfermeiros presentes nas regiões mais favorecidas, um não foi formado no país onde atua, demonstrando que os países de alta renda defrontam-se com uma fragilidade em seu sistema de formação de enfermeiros, obrigando-os a recrutar profissionais de países vizinhos ou de outras regiões que já se encontram desguarnecidas. ${ }^{1}$ Nesse sentido, a cooperação entre os países emerge como importante estratégia para lidar com as mencionadas desigualdades no contexto da formação de enfermeiros, número de profissionais e condições de trabalho dentro e entre países.

Nesse sentido, a Cooperação Técnica entre Países (CTP) pode resultar em desenvolvimento, fortalecimento, intercâmbio de conhecimentos técnicos e científicos, além da formação de recursos humanos. ${ }^{2}$ A cooperação técnica engloba planejamento, desenvolvimento e implementação de ações coordenadas visando o atingimento de maiores níveis de desenvolvimento em áreas chave, como a saúde. Os objetivos da cooperação técnica internacional caminham em direção à modernização, trazendo consigo a proposta de nivelar a qualidade de vida, por meio de ações conjuntas em áreas distintas, idealizadas e implementadas por diferentes atores internacionais. Em sintonia com essa complexa missão, é fundamental que os Estados reconheçam as bases culturais e sociais de cada nação, transformando eixos econômicos e sociais e impactando na vida de uma sociedade de forma benéfica. ${ }^{3}$

Nos últimos anos, a relevância da cooperação internacional entre os Estados tem sido perceptível, transformando a cooperação técnica em eixo norteador da expansão da política externa. Nesse contexto, o Brasil vem desempenhando um papel relevante, especialmente no âmbito da cooperação horizontal e na área da saúde. Esse direcionamento da cooperação resultou em importantes benefícios para a política externa brasileira, especialmente considerando a agenda de saúde global e o despertar para as desigualdades de saúde dentro e fora dos países. ${ }^{4,5}$

Dessa forma, o setor saúde tem se destacado com a cooperação sul-sul, ou cooperação horizontal, implementada entre países em desenvolvimento, que nasce em meados de 1970, em decorrência de movimentos internacionais por uma nova divisão do trabalho internacional, que englobasse os interesses específicos dos países periféricos. Essa cooperação é evidenciada pela contínua troca de experiências, compartilhamento de conhecimento, aprendizado e resultados, além de assumir responsabilidades conjuntas em âmbito nacional e internacional, moldadas por diretrizes estratégicas e com base em princípios democráticos. ${ }^{5}$

A cooperação horizontal em saúde proporciona a disseminação da ciência e tecnologia entre países, buscando fortalecer políticas sanitárias em todas os âmbitos e instâncias territoriais. ${ }^{6}$ Nesse contexto, o Brasil vem desempenhando importante papel na Cooperação Sul-Sul, por meio de cooperação técnica em saúde na América do Sul e com países africanos, pautados na horizontalidade e solidariedade. ${ }^{7}$

Com perspectiva duradoura, a cooperação técnica visa o planejamento e desenvolvimento de ações conjuntas de caráter técnico para beneficiar uma área específica, como a saúde. É bilateral ou multilateral, com recursos financeiros e humanos provenientes de todos os envolvidos no projeto. Este tipo de cooperação se diferencia da ajuda ou assistência, normalmente de caráter humanitário, que engloba ações de cooperação de âmbito unilateral em casos de emergências internacionais.

No contexto da Enfermagem, a cooperação técnica entre - Brasil e países africanos é bastante significativa, visando especialmente a formação de enfermeiros. Dessa forma, nas décadas de 1980 e 1990, as universidades públicas brasileiras receberam considerável número de estudantes provenientes da África, muitos deles cursando Enfermagem. Pode-se afirmar que os primeiros Bacharéis e Licenciados em Enfermagem de Angola e Moçambique foram formados no Brasil.

Considerando seu histórico de cooperação horizontal do Brasil com países africanos de língua portuguesa, este estudo apresenta como objetivos caracterizar os desafios vivenciados pela Enfermagem nos países africanos de língua portuguesa e discutir as potencialidades da cooperação internacional neste contexto.

\section{MÉTODO}

Trata-se de estudo qualitativo, descritivo, cujos dados foram obtidos por meio de entrevista semiestruturada com enfermeiros de países africanos de língua portuguesa, participantes do workshop: "A Enfermagem no Brasil e nos países Africanos de Língua Portuguesa". Participaram do estudo enfermeiros de Angola, Cabo Verde, Guiné Bissau, Moçambique e São Tomé e Príncipe. Todos os enfermeiros participantes do Workshop, organizado em conjunto com o Ministério da Saúde do Brasil e a Representação no Brasil da Organização Pan-Americana da Saúde, foram convidados a participar deste estudo. Os participantes do Workshop eram enfermeiros de países africanos de língua portuguesa que possuíam parcerias com instituições de ensino e serviços no Brasil. A coleta de dados ocorreu sob a responsabilidade de três dos autores que participaram, também, da organização do Workshop.

Para a coleta de dados foi utilizado o formulário elaborado pelos autores, estruturado a partir dos objetivos do estudo que considerou, nesse processo o conceito e os elementos da cooperação internacional horizontal em saúde. A coleta ocorreu ao final do workshop, em São Paulo, durante evento que reuniu os participantes da pesquisa. 
Para o tratamento dos dados aplicou-se a técnica de análise de conteúdo por categorias temáticas. Nesse sentido, os dados foram codificados em unidades de registro e agregados em unidades de significado, categorizados por similaridades. A análise ocorreu reiteradas vezes por dois pesquisadores independentes; caso houvesse divergência na análise, um terceiro pesquisador poderia ser acionado; contudo, não foi necessário. As unidades de significado foram agrupadas em subcategorias e, posteriormente, em categorias. Sendo assim, as categorias foram construídas conforme os temas que emergiram do texto, identificando-se o que tinham em comum para seu agrupamento. ${ }^{8,9}$ Os participantes foram identificados pela letra $P$, seguida por uma sequência crescente de números cardinais, conforme a ordem de realização das entrevistas.

Para a realização da pesquisa, todos os aspectos éticos e legais, referentes ao desenvolvimento de pesquisa com seres humanos, foram cumpridos. Em assim sendo, o projeto foi devidamente apreciado e aprovado por Comitê de Ética em Pesquisa com Seres Humanos (Of CEP-EERP/USP - 157/2011. Protocolo n 1290/2011).

\section{RESULTADOS}

Participaram do workshop nove enfermeiros, majoritariamente do sexo feminino, com idade entre 31 a 54 anos, sendo um de Angola, três de Cabo Verde, dois de Guiné-Bissau, dois de Moçambique e um de São Tomé e Príncipe. Em termos de formação, apenas dois participantes possuíam mestrado/ doutorado, sendo que $66,6 \%$ ocupavam funções acadêmicas. Ao serem questionadas sobre o conhecimento de algum projeto de cooperação (ajuda ou assistência técnica) desenvolvido em seu país, em cooperação com algum país estrangeiro na área da Enfermagem, 55,5\% responderam ter conhecimento-55,5\% de cooperação técnica ou $44,4 \%$ de ajuda ou assistência. Os participantes referiram, ainda, que muitos projetos, antes vigentes, estavam estagnados; $22,2 \%$ responderam haver dificuldades logísticas e 44,4\% declararam observar dificuldades relacionadas à falta de recursos financeiros para o desenvolvimento de projetos.

Da análise temática emergiram duas categorias, descritas a seguir.

\section{Enfermagem dos países africanos de língua portuguesa: adversidades e similaridades com a Enfermagem brasileira}

De acordo com os participantes, as dificuldades encontradas eram eminentemente provenientes de desmotivação, como reflexo de sua "autonomia roubada", definição pinçada da essência dos depoimentos. Para eles, as dificuldades vivenciadas paralisam toda a estrutura humana e funcional. Caracterizam-se pela dificuldade de trabalho com a equipe multidisciplinar, especialmente com os médicos, resultando na falta de reconhecimento de sua autonomia profissional.

[...] Dificuldade no trabalho na equipe multidisciplinar e o reconhecimento da qualidade dos profissionais $(\mathrm{P} 1)$.
Dificuldade na inserção no sistema Nacional de Saúde como profissional autônomo e com poder decisório. Falta de autonomia profissional por forte intervenção de profissionais da área médica (P 2).

[...] Falta de autonomia, que é roubada por outros profissionais [...] pouca remuneração que implica falta de motivação (P4).

A desvalorização da contribuição da Enfermagem pela equipe e lideranças da área da saúde refletidas em remuneração baixa, ausência de progressão no nível acadêmico e de promoção dos enfermeiros, falta de autonomia para o exercício da profissão foram pontos centrais no discurso dos participantes.

Pouca integração de enfermeiros em atividades de saúde (P2).

[...] A não implementação da carreira (P9).

[...] Impedimento de progressão e de promoção dos enfermeiros (P 6).

[...] Não participação dos enfermeiros em atividades de saúde por falta de autorização do Ministério de Saúde (P4).

Os principais entraves para acesso à educação continuada e ausência de investimentos na formação especializada, como cursos especialização, continuidade na pós-graduação, déficit de instituições de ensino superior para formação específica de enfermagem foram relatados também:

As dificuldades são da seguinte ordem: nas áreas de formação dos enfermeiros e parteiras sobre licenciatura em enfermagem/obstétrica, inexistência do curso da especialização/enfermagem (P8).

[...] Falta de Escolas Superiores em Enfermagem (P 6).

[...] Número baixo de profissionais com nível superior e continuidade na pós-graduação (P1).

Os resultados da categoria supracitada revelam, portanto, dificuldades para o exercício da profissional de Enfermagem com autonomia necessária ao amplo desempenho de suas competências. Essa realidade apresenta, porém, elementos que tangenciam a realidade da Enfermagem brasileira. Apesar disso, a segunda categoria revela possibilidades de fortalecimento da Enfermagem, em uma perspectiva de redes que articulem e conectem fortalezas e possibilidades para o desenvolvimento da profissão nesses diferentes países.

\section{Potencial de colaboração entre os países de língua portuguesa}

As alternativas de colaboração proporcionam investimento no capital humano da Enfermagem, a partir de formação técnica e humana e, principalmente, a valorização da especificidade da contribuição dessa profissão e ciência. Além disso, estimula o 
desenvolvimento de parcerias em pesquisa e para formação de enfermeiros qualificados por meio da colaboração entre universidades e organizações.

Intercâmbio em pesquisa científica. Intercâmbio e formação do pessoal de enfermagem em nível superior incluindo pós-graduação (mestrado e doutorado). Abertura de escola superior de enfermagem acadêmica regional. Fortalecimento de intercâmbio em busca de fontes financiadoras para o desenvolvimento de enfermagem nos países (P 6).

Mais comunicação das atividades entre os países. Participação em mais eventos internacionais onde todos participem. - Troca de experiências entre equipes de enfermagem dos países. - Supervisão sobre o perfil do profissional em vários países (P1).

Intercâmbio de conhecimento e vivências que poderiam melhorar as atividades. Parcerias entre países representantes para desenvolvimento de alguns projetos, visto que temos muitos problemas e potencialidades em comum. Cooperação em termos de formações e participação em atividades desenvolvidas na Organização Pan-Americana de Saúde (OPAS) (P 2).

Criação da Ordem de Enfermeiros nos PALOP. Criação de instituições de ensino superior para os enfermeiros dos PALOP. Criação de projetos/centros de pesquisa conjunta. Fortalecimento das bibliotecas móveis. Organização de jornadas científicas de forma rotativa nos PALOP (P5).

As potencialidades podem ser adquiridas com o desenvolvimento de conhecimentos e experiências comuns, no âmbito da ciência, por meio de investigação em Enfermagem baseada em evidência.

Cooperação em formação. Intercâmbio trocando experiências da prática dos profissionais que na área de gestão dos programas de saúde ou cuidados primários, docência e pesquisa. Ajuda na busca de financiamentos para os projetos de desenvolvimento (P 6).

Parcerias para especializações e pós-graduação em geral. Divulgar melhor o nosso país e saber mais sobre os outros países que participarem. Mais conhecimentos: condições para tornar melhores profissionais (P 4).

\section{DISCUSSÃO}

As dificuldades encontradas pela Enfermagem, em relação à falta de autonomia, como reflexo da falta de reconhecimento de seu valor e importância para o setor de saúde são características presentes em diversos países, de praticamente todas as regiões. Desafios enfrentados, por exemplo, pelos enfermeiros na Índia demonstraram a existência de forte desajuste no vínculo entre as demais categorias profissionais, caracterizando um grande desafio para um relacionamento produtivo entre essas equipes. Nesse sentido, os profissionais de enfermagem carregam o peso de suportarem as dores de estarem na linha de frente nos sistemas de saúde e não serem reconhecidos. ${ }^{10}$

São frequentes os fatores que geram exaustão física e emocional e interferem na motivação do enfermeiro no ambiente hospitalar, indicando a falta de autonomia, assim como a influência da autoridade do médico sobre o seu trabalho, hierarquização, sobrecarga de trabalho, escassez de recursos humanos e insumos, geradores de exaustão física e emocional. ${ }^{11}$ Esses desgastes impactam diretamente em suas vidas e no seu processo de trabalho, levando à desmotivação constatada nas falas dos participantes desta pesquisa. Nessa perspectiva, alguns participantes mencionam que se sentem com sua "autonomia roubada" pela estrutura vigente e também considerando o relacionamento com outros membros da equipe de saúde.

Esse cenário de dificuldades no reconhecimento e na (in) visibilidade do trabalho de enfermagem tem sido reportado em vários estudos; há evidências de que quando o profissional de enfermagem não é valorizado, a expressão de sua identidade profissional é perdida, descaracterizando-se a importância de sua atuação profissional. ${ }^{12-15}$ Os resultados do presente estudo apontam para a clara desmotivação dos profissionais de enfermagem participantes da pesquisa, como consequência do sentimento de desvalorização profissional.

O preconceito vivenciado pela enfermagem é analisado nas relações sociais que corroboram a vivência da exclusão do enfermeiro, que muitas vezes aceita esta condição por estar inserido num contexto social em que esse conceito limitado está cristalizado. Nessas condições, o enfermeiro desvaloriza a si próprio e aos outros, com baixa autoestima e até aversão aos próprios colegas. ${ }^{16}$ Muitos conflitos vivenciados pela enfermagem decorrem do predomínio do modelo biomédico, em que o profissional não consegue exercer a sua profissão de forma autônoma, sendo subordinado às ordens/aprovações que os médicos impõem. ${ }^{17}$

A precarização do mercado de trabalho de enfermagem foi registrada em estudo transversal realizado entre 2005 e 2018 em 32 estados do México, tornando-se crescente como consequência também de grandes reformas trabalhistas, afetando os profissionais de enfermagem, na maioria assalariados. Naquele país, houve um aumento de $8,6 \%$ de admissões de enfermeiros sem contrato, $53,4 \%$ com baixos salários e outra parte dessa população está atuando em modalidade subempregada. ${ }^{18}$

Por outro lado, uma integração interprofissional nas equipes de saúde ocorre em alguns países. Estudo realizado com enfermeiros da Inglaterra revelou que alguns enfermeiros conseguem exercer sua autonomia por meio do trabalho em equipe, em suas práticas diárias compartilhando seus conhecimentos e habilidades. Dessa forma, enfermeiros relataram que a hierarquia dos sistemas de saúde, assim como as políticas e diretrizes são determinantes para sua atuação de forma autônoma, ${ }^{19}$ primordial para sua motivação e satisfação no contexto do ambiente de trabalho. 
Já no campo da formação profissional, estudo com gestores de instituições públicas e privadas no estado do nordeste brasileiro demonstrou que embora tenha havido ao longo do anos expansão de instituições privadas voltadas para a formação de enfermeiros, não foi observado crescimento de contratação de profissionais de saúde nas instituições de saúde, resultando em um grande número de profissionais desempregados. ${ }^{20}$

Em Portugal, foi realizado levantamento da força de trabalho da enfermagem nos últimos 40 anos - desde a criação do Serviço Nacional de Saúde, constatando-se avanços no conhecimento técnico e científico nesses anos investigados. Nota-se mudança no quadro educacional voltado para a formação especializada dos enfermeiros. ${ }^{21}$

Apesar das dificuldades relatadas pelos participantes, a colaboração entre enfermeiros de outros países, especialmente de língua portuguesa, foi apontada como estratégia alternativa para seu fortalecimento.

Ressalta-se, nesse sentido, o potencial de colaboração entre os países de língua portuguesa, constituídos pela Comunidade de Países de Língua Oficial Portuguesa (CPLP). Mas, no que tange especialmente ao continente africano, considerando aspectos identitários comuns aos Países Africanos de Língua Portuguesa (PALOP) são sugeridas iniciativas como a organização de mais eventos para o intercâmbio de vivências e até a criação de uma Ordem de Enfermeiros nos PALOP, ou outra iniciativa integradora da enfermagem nesses países, chancelada pelos respectivos Ministérios da Saúde e das Relações Exteriores de cada país, para que programas comuns possam ser desenvolvidos com frequência e na medida das necessidades apontadas pelos próprios profissionais de enfermagem e consensuadas nesta instância integradora. Desse modo, identificada a demanda, acionam-se instituições do Brasil e de Portugal que possam desenvolver juntos e sequencialmente cada programação estratégica nas diferentes temáticas. Se no século XX havia uma forte limitação pelas distâncias geográficas e pela frágil infraestrutura digital que favorecesse pontes entre a enfermagem dos países integrantes dos PALOP, ${ }^{22,23}$ nesta segunda década do século XXI não podemos mais aceitar que não nos valhamos do avanço da internet e de aplicativos nos dispositivos móveis para avançarmos em busca de integração pelo conhecimento.

Como já ressaltado na literatura de enfermagem, importa reconhecer a tendência da nova ordem pós moderna de que por meio da tecnologia é possível fomentar o desenvolvimento dos recursos humanos - "o bem mais valioso de que as sociedades dispõem para cuidar, promover e educar em saúde. Não adianta técnica, recursos financeiros, poder, se não houver sistemas de saúde que funcionem, que dêem a garantia de acessibilidade gratuita com produtividade, qualidade, equidade e justiça social". ${ }^{24}$ Nessa perspectiva, o conhecimento representa o poder de que os participantes da presente pesquisa mais se ressentem para resgatar e fazer valer sua autonomia.

A atual política de saúde digital, salientada pela Assembleia Mundial de Saúde em 2018, aprovou estratégia de promoção de colaboração global para impulsionar a transferência de conhecimento por meios digitais. Esta proposta advoga o alinhamento de países e colaboradores para que, em conjunto, explorem oportunidades globais e atinjam objetivos usando tecnologias digitais para melhorar saúde e possibilitar cobertura universal - o cerne dos Objetivos de Desenvolvimento Sustentável relativos à saúde. ${ }^{25} \mathrm{~A}$ cobertura universal de saúde é o maior desafio a ser enfrentado pelos sistemas de saúde em todo o mundo, especialmente nos países em desenvolvimento ; nesse desafio reside um potencial de visibilidade e valorização dos enfermeiros por meio de suas intervenções consolidando os princípios dessa política de saúde. ${ }^{26}$

Nesse cenário, todos os países devem avançar seu investimento em tecnologias digitais e saúde, de modo que agora seja possível aproximarmos os países falantes de português e, de fato, colocarmos em curso programações que há muito se apresentam como um desejo comum, mas antes impedidas pela barreira tecnológica presente nos países africanos. Portanto, é fundamental que entidades e lideranças de enfermagem envidem esforços para técnica e politicamente conseguirmos avançar propostas de compartilhamento solidário de conteúdos, dados, experiências, desafios, barreiras e êxitos conquistados pela enfermagem. Ações afirmativas terão um poder gerador de autoconfiança e auto-realização, reforçando o propósito de lutar pela autonomia que enfermeiros africanos sentem e definem como roubada, e resgatá-la pelo poder do conhecimento atualizado. $\mathrm{Na}$ era atual de apelo intenso por comunicações sociais instantâneas, e também por saúde digital, não faltam veículos e formatos que permitam a operacionalização de programas projetados e capazes de solucionar o distanciamento dos recursos humanos da área de enfermagem que irmanam-se pelo mesmo idioma nos continentes africano, americano e europeu. Dessa forma, por meio desta ponte é possível promover igualdade..$^{25}$

E em alinhamento com a janela de oportunidade aberta pelo movimento Nursing Now no Ano Internacional da Enfermagem, cresce a conscientização global sobre o valor do investimento na saúde como um bem público e a representatividade e o potencial precioso dos enfermeiros e obstetrizes na melhoria da saúde, na criação da igualdade de gênero e no fortalecimento das economias. Nessa onda de visibilidade e valorização, cada vez mais enfermeiros encorajam-se para "romper o silêncio" e apropriarem-se do seu direito de propagar sua voz nas mesas decisórias e contribuir na definição de políticas de saúde. ${ }^{27-29}$ Enfim, lições exitosas mostram que em havendo postura política e estratégica de instituições e de lideranças capazes de desenvolver e acentuar intercâmbio de experiências na horizontalidade da cooperação Sul-Sul com os PALOP, com instrumentos que melhor se adequem a cada necessidade e potencialidades das instituições cooperantes, poderemos superar um desafio que há décadas vimos apontando como urgente; quiçá, que com a vigência da estratégia de saúde digital se promova cooperação global, de modo que o esforço sul-sul receba aportes do sentido norte-sul e que, somados, ganhem e sejam indutores de fortalecimento, agilidade, equidade e solidariedade no engrandecimento dos recursos humanos de enfermagem. E que, valendo-se do 
movimento vigoroso em prol da valorização da Enfermagem e Obstetrícia, ganhem visibilidade $\mathrm{e}^{13,14}$ em seu país.

\section{CONCLUSÃO}

O presente estudo caracterizou os crescentes desafios enfrentados pela enfermagem em países africanos de língua portuguesa e a expectativa de enfermeiros provenientes desses países com relação ao potencial de cooperação internacional, envolvendo especificamente o Brasil.

Dessa forma, as falas sobre as dificuldades relacionadas aos processos de trabalho vivenciados, falta de autonomia e de estrutura humana e física emergiram como um grande desafio a ser enfrentado pelos enfermeiros em suas atividades cotidianas. Para lidar com esse contexto adverso, os participantes reforçam o histórico de colaboração existente, no âmbito da cooperação sul-sul na área da saúde, envolvendo o Brasil e esses países. Os dados demonstram o potencial, a partir da perspectiva dos participantes da pesquisa, da cooperação entre os Países Africanos de Língua Oficial Portuguesa no que tange à colaboração na formação técnica e humana, intercâmbio em pesquisa científica, formação do pessoal de enfermagem em nível superior incluindo pós-graduação, visando-se fomentar um desenvolvimento sustentável para a enfermagem.

Nesse cenário, ressalta-se a utilização de tecnologias digitais como recursos fundamentais para a construção de colaborações duradouras, especialmente para a formação e capacitação de recursos humanos entre os Países Africanos de Língua Oficial Portuguesa.

Considera-se como limitação a amostra de conveniência centrada em participantes de um Workshop. Contudo, apesar de ter envolvido um número reduzido de enfermeiros dos cinco países, o estudo aponta para direcionamentos importantes visando o fortalecimento da colaboração em enfermagem entre os países envolvidos. Sendo assim, sugere-se o desenvolvimento de estudos que aprofundem esta temática e avaliem de forma sistematizada diferentes experiências de cooperação internacional na área da saúde e com foco na enfermagem.

A valorização das relações de cooperação técnica internacional em saúde é fundamental para o aperfeiçoamento da prática dos enfermeiros. No caso dos profissionais enfermeiros de países africanos de língua portuguesa, a cooperação foi fundamental para a sua formação profissional e atua como instrumento de apoio para o seu fortalecimento e aperfeiçoamento. A cooperação internacional pode ser considerada estratégica para que enfermeiros de países africanos de língua portuguesa possam lidar com os constantes desafios vivenciados em seus cenários de atuação.

\section{AGRADECIMENTOS}

Os autores reconhecem o mérito da Profa. Dra. Maria Auxiliadora Trevizan na organização e desenvolvimento do Workshop: "A Enfermagem no Brasil e nos países Africanos de Língua Portuguesa".

\section{CONTRIBUIÇÕES DOS AUTORES}

Desenho do estudo. Carla Aparecida Arena Ventura.

Coleta ou produção dos dados. Carla Aparecida Arena Ventura. Leila Maria Marchi-Alves. Isabel Amélia Costa Mendes.

Análise de dados. Carla Aparecida Arena Ventura. Isabel Amélia Costa Mendes. Ítalo Rodolfo Silva. Emerson Willian Santos Almeida. Simone de Godoy.

Interpretação dos resultados. Isabel Amélia Costa Mendes. Ítalo Rodolfo Silva. Leila Maria Marchi-Alves. Emerson Willian Santos Almeida. Simone de Godoy.

Redação e revisão crítica do manuscrito. Carla Aparecida Arena Ventura. Isabel Amélia Costa Mendes. Ítalo Rodolfo Silva Leila Maria Marchi-Alves. Emerson Willian Santos Almeida. Simone de Godoy.

Aprovação da versão final do artigo. Carla Aparecida Arena Ventura. Isabel Amélia Costa Mendes. Ítalo Rodolfo Silva. Leila Maria Marchi-Alves. Emerson Willian Santos Almeida. Simone de Godoy.

Responsabilidade por todos os aspectos do conteúdo e a integridade do artigo publicado. Carla Aparecida Arena Ventura. Isabel Amélia Costa Mendes. Ítalo Rodolfo Silva. Leila Maria Marchi-Alves. Emerson Willian Santos Almeida. Simone de Godoy.

\section{EDITOR ASSOCIADO}

\author{
Cristina Rosa Baixinho
}

\section{REFERÊNCIAS}

1. World Health Organization. State of the world's nursing 2020: investing in education, jobs and leadership. Geneva: WHO; 2020.

2. Organização Pan-Americana da Saúde. Organização Mundial da Saúde. Cooperação técnica entre países na região. Washington: OPAS/OMS 2005.

3. Satto E. Cooperação internacional: uma componente essencial das relações internacionais. R. Eletr. de Com. Inf. Inov. Saúde. 2010;4(1):4657. http://dx.doi.org/10.3395/reciis.v4i1.345pt.

4. Mazzaroppi E. Evolução histórico-conceitual da Cooperação Técnica Internacional Brasileira em Saúde. Rev Eletr. Com. Inf. Inov. Saúde. 2016;10(3):1-11. http://dx.doi.org/10.29397/reciis.v10i3.1087.

5. Almeida C, Campos RP, Buss P, Ferreira JR, Fonseca LE. A concepção brasileira de "cooperação Sul-Sul estruturante em saúde". R. Eletr. de Com. Inf. Inov. Saúde. 2010;4(1):25-35

6. Organização Pan-Americana da Saúde. Organização Mundial da Saúde. Cooperação para o desenvolvimento da saúde nas Américas. Washington: OPAS/OMS; 2013.

7. Santos RF, Cerqueira MR. South-South Cooperation: Brazilian experiences in South America and Africa. Hist. cienc. saude, 2015;22(1):23-47. http:// dx.doi.org/10.1590/S0104-59702015000100003.

8. Bardin L. Análise de conteúdo. Lisboa: Edições 70; 1995.

9. Caregnato RCA, Mutti R. Pesquisa qualitativa: análise de discurso versus análise de conteúdo. Texto Contexto Enferm. 2006;15(4):67984. http://dx.doi.org/10.1590/S0104-07072006000400017.

10. Chhugani M, James MM. Challenges faced by nurses in india-the major workforce of the healthcare system. Nurse Care Open Acces J. 2017;2(4):112-4. http://dx.doi.org/10.15406/ncoaj.2017.02.00045.

11. Bonfada MS, Pinno C, Camponogara S. Potentialities and limits of nursing autonomy in a hospital environment. J Nurs UFPE online. 2018; 12(8):223546. https://doi.org/10.5205/1981-8963-v12i8a234915p2235-2246-2018. 
12. Silva AR, Padilha MICS, Backes VMS, Carvalho JB. Professional nursing identity: a perspective through the brazilian printed media lenses. Esc Anna Nery. 2018;22(4):e20180182. http://dx.doi.org/10.1590/21779465-ean-2018-0182.

13. Salvage J, Stilwell B. Breaking the silence: a new story of nursing. J Clin Nurs. 2018;27(7-8):1301-3. http://dx.doi.org/10.1111/jocn.14306. PMid:29430758.

14. Mitchell PH. Nursing science and health policy- opportunities in the year of the nurse and midwife. Int Nurs Rev. 2020;67(1):1-3. http://dx.doi. org/10.1111/inr.12577. PMid:32083729.

15. The Lancet. The status of nursing and midwifery in the world [editorial]. Lancet. 2020;395(10231):1167. http://dx.doi.org/10.1016/S01406736(20)30821-7.

16. Seago JA. Autonomìa: ¿unametapara la pràctica hospitalaria de enfermeria? Aquichan. 2006;6(1):92-103.

17. Melo CMM, Florentino TC, Mascarenhas NB, Macedo KS, Silva MC Mascarenhas SN. Professional autonomy of the nurse: some reflections. Esc Anna Nery. 2016;20(4):e20160085. http://dx.doi.org/10.5935/14148145.20160085

18. Aristizabal P, Nigenda G, Servan-Mori E. The precarization of the Mexican nursing labor market: a repeated cross-sectional analysis for the period 2005-2018. Hum Resour Health. 2019;17(1):1-9. http:// dx.doi.org/10.1186/s12960-019-0417-x. PMid:31753033.

19. Oshodi TO, Bruneau B, Crockett R, Kinchington F, Nayar S, West E. Registered nurses' perceptions and experiences of autonomy: a descriptive phenomenological study. BMC Nurs. 2019;18:51. http:// dx.doi.org/10.1186/s12912-019-0378-3. PMid:31695577.

20. Oliveira JSA, Pires DEP, Alvarez AM, Sena RR, Medeiros SM, Andrade $\mathrm{SR}$. Trends in the job market of nurses in the view of managers. Rev Bras Enferm. 2018;71(1):148-55. http://dx.doi.org/10.1590/0034-71672016-0103. PMid:29324957.
21. Fronteira I, Jesus EH, Dussault G. Nursing in Portugal in the Nationa Health Service at 40. Ciênc. saúde coletiva. 2020;25(1):273-82. http:// dx.doi.org/10.1590/1413-81232020251.28482019.

22. Costa Mendes IA, Marchi-Alves LM, Mazzo A, Nogueira MS, Trevizan MA, de Godoy $S$ et al. Healthcare context and nursing workforce in a main city of Angola. Int Nurs Rev. 2013;60(1):37-44. http://dx.doi. org/10.1111/j.1466-7657.2012.01039.x. PMid:23406235.

23. Marchi-Alves LM, Ventura CA, Trevizan MA, Mazzo A, de Godoy S, Mendes IA. Challenges for nursing education in Angola: the perception of nurse leaders affiliated with professional education institutions. Hum Resour Health. 2013;11:33. http://dx.doi.org/10.1186/1478-4491-11-33. PMid:23866740.

24. Trevizan MA, Mendes IAC, Mazzo A, Ventura CAA. Investment in nursing human assets: education and minds of the future. Rev Lat Am Enfermagem. 2010 jun;18(3):467-71. http://dx.doi.org/10.1590/S010411692010000300024. PMid:20721438.

25. World Health Organization. Draft global strategy on digital health 2020 2024. Geneva: WHO; 2020

26. Mendes IA, Ventura CA, Trevizan MA, Marchi-Alves LM, de SouzaJunior VD. Education, leadership and partnerships: nursing potential for Universal Health Coverage. Rev Lat Am Enfermagem. 2016;24:e2673. http://dx.doi.org/10.1590/1518-8345.1092.2673. PMid:26959333.

27. Salvage J, White J. Nosso futuro é global: liderança em enfermagem e saúde global. Rev Lat Am Enfermagem. 2020;28:e3339. http://dx.doi org/10.1590/1518-8345.4542.3339. PMid:32876292.

28. Mendes IAC, Trevizan MA. In the absence of light, the Nursing Now lighthouse illuminates the future. Rev Lat Am Enfermagem. 2020;28:e3356. http://dx.doi.org/10.1590/1518-8345.0000.3356. PMid:33084771.

29. Benton $D$, Shaffer $F$. How the nursing profession can contribute to the sustainable development goals. Nurs Manag (Harrow). 2016;23(7):2934. http://dx.doi.org/10.7748/nm.2016.e1534. PMid:27800732. 